\title{
Klarer Zusammenhang bei Typ-2-Diabetikern entdeckt
}

\begin{abstract}
Die Auswertung von GAPP2 ${ }^{\text {TM }}$, einer Befragung von Typ-2-Diabetikern und Ärzten, hat ergeben, dass 46\% der Patienten ihr Basalinsulin in den 30 Tagen vor der Erhebung einmal ausgelassen bzw. nicht zum richtigen Zeitpunkt injiziert haben. Patienten, die eine Dosis ihres Basalinsulins versäumt hatten, berichteten außerdem besonders häufig von einer selbst behandelten Hypoglykämie.
\end{abstract}

Im Rahmen der GAPP2 ${ }^{\text {Tm}}$-Beobachtungsstudie (Global Attitudes of Patients and Physicians) wurden zwischen Januar und März 2012 über 3000 Menschen mit Typ2-Diabetes, die ein modernes Basalinsulin verwenden, sowie etwa 1650 Therapeuten in sechs Ländern (USA, Kanada,

Brod M et al. GAPP2TM: Global survey finds in the last month one in four type 2 diabetes patients do not take basal insulin as prescribed and over a third suffer hypoglycaemia. Diabetes 61 (Suppl. 1A) 2012: 27-LB, 5579
Japan, Deutschland, Vereinigtes Königreich, Dänemark) befragt. Die ersten Ergebnisse des von Novo Nordisk initiierten Surveys wurden bei der 72. Jahrestagung der American Diabetes Association (ADA) in Philadelphia präsentiert.

Dabei zeigte sich, dass Dosierungsunregelmäßigkeiten bei Menschen mit Typ2-Diabetes, die Insulin erhalten, nicht ungewöhnlich sind. In den 30 Tagen vor der Befragung haben 22\% eine Dosis ihres Basalinsulins ausgelassen, 24\% die Dosis um mehr als zwei Stunden versetzt appliziert und 14\% eine zu geringe Dosis injiziert. Es gab außerdem eine Korrelation zwischen Dosierungsunregelmäßigkeiten und Hypoglykämien: Bei jenen, die in den 30 Tagen vor der Befragung eine Dosis ihres Basalinsulins versäumt hatten, waren selbst behandelte Unterzuckerungen im gleichen Zeitraum signifikant wahrscheinlicher (41\% vs. $34 \%$ ).

„Das Besondere an GAPP2 ${ }^{\mathrm{Tm}}$ ist, dass erstmals Dosierungsunregelmäßigkeiten und selbst behandelte Hypoglykämien gleichzeitig in einer Person betrachtet wurden, und dass es so möglich war, eine Verbindung zwischen den Problemen herzustellen“, erklärte Dr. Meryl Brod, Studienleiterin aus San Francisco, USA. „Um das Management von Menschen mit Typ-2-Diabetes zu verbessern, sind neben einer besseren Schulung von Ärzten und Patienten auch bessere Insuline nötig. Diese sollten das Risiko einer Hypoglykämie vermindern und flexibler in Bezug auf das Zeitfenster der Applikation sein.“

Bericht: Monika Walter, München

Quelle: Exklusive GAPP2 ${ }^{\text {TM}}{ }^{-i n t e r v i e w s ~ m i t ~ M e r y l ~}$ Brod anlässlich des ADA-Kongresses, 10.6.2012 in Philadelphia, USA, Veranstalter: Novo Nordisk

\section{Register zur Erfolgskontrolle gestartet}

\begin{abstract}
Nach dem Motto "Ohne Register keinen Durchblick" soll das Register DIALOGUE erstmals überprüfen, wie erfolgreich hypertensive Typ-2Diabetiker behandelt werden. DIALOGUE startete im Sommer 2012 in etwa 600 deutschen Arztpraxen.
\end{abstract}

Für Prof. Diethelm Tschöpe aus Bad Oeynhausen, Prof. Anselm Gitt aus Ludwigshafen und Prof. Roland E. Schmieder aus Erlangen, Mitglieder des Lenkungsausschusses, ist „ein Register wie DIALOGUE längst überfällig. Den größten Stellenwert wird die individuelle Zielwerterreichung der hypertonen Diabetiker besitzen: „Die idealen Blutzuckerund Blutdruckwerte werden vom behandelnden Arzt auf jeden Patienten angepasst, abhängig von Lebensalter, Komorbiditäten oder Lebenserwartung." Erste Patienten wurden im Sommer rekrutiert.
Interimsanalysen sind nach sechs und zwölf Monaten geplant. Nach optionalen Follow-ups nach 36 bzw. 48 Monaten ist der Bericht über die klinischen Daten im April 2016 zu erwarten.

Rund 90\% der Typ-2-Diabetiker leiden gleichzeitig an Hypertonie, was auf gemeinsame Risikofaktoren zurückzuführen ist. Schon Diabetes allein erhöht das Risiko für Folgeerkrankungen wie Schlaganfall oder Lungenödeme. Ein Patient mit Typ-2-Diabetes und Hypertonie hat ein bis zu vierfach erhöhtes kardiovaskuläres Morbiditäts- und Mortalitäts- risiko im Vergleich zu einem Nichtdiabetiker mit Bluthochdruck.

Mit dem DIALOGUE-Register plant Novartis Pharma für das Jahr 2012 die Erfassung des individuellen Behandlungserfolges verschiedener antidiabetischer und antihypertensiver Therapien sowie deren Kombination in Bezug auf die individuelle Zielwerterreichung nach 24 Monaten.

Mit 10000 hypertonen Typ-2-Diabetikern wird DIALOGUE das weltweit erste Register sein, das die Ergebnisse einer kombinierten antidiabetischen und antihypertensiven Therapie auswertet.

Verglichen werden in DIALOGUE inkretinbasierte und nicht inkretinbasierte Diabetestherapien sowie RAAS-basierte und nicht RAAS-basierte Hypertonietherapien.

Bericht: Dr. Jochen Aumiller, München

Quelle: Fachpressekonferenz „Orale Antidiabetika - welchen Zusatznutzen bietet eine moderne Diabetestherapie?", DDG-Jahrestagung, 16.5. 2012 in Stuttgart, Veranstalter: Novartis Pharma 